\title{
Analytical Solutions of Some Fully Developed Flows of Couple Stress Fluid between Concentric Cylinders with Slip Boundary Conditions
}

\author{
M. Devakar, ${ }^{1}$ D. Sreenivasu, ${ }^{2}$ and B. Shankar ${ }^{3}$ \\ ${ }^{1}$ Department of Mathematics, Visvesvaraya National Institute of Technology, Nagpur 440 010, India \\ ${ }^{2}$ Department of Mathematics, CVR College of Engineering, Hyderabad 501 510, India \\ ${ }^{3}$ Department of Mathematics, Osmania University, Hyderabad 500 007, India
}

Correspondence should be addressed to M. Devakar; m_devakar@yahoo.co.in

Received 28 January 2014; Revised 13 May 2014; Accepted 28 May 2014; Published 17 June 2014

Academic Editor: Viktor Popov

Copyright (C) 2014 M. Devakar et al. This is an open access article distributed under the Creative Commons Attribution License, which permits unrestricted use, distribution, and reproduction in any medium, provided the original work is properly cited.

\begin{abstract}
We establish, in this paper, the closed form analytical solutions of steady fully developed flows of couple stress fluid between two concentric cylinders, generated due to the constant pressure gradient or the translatory motion of the outer cylinder or both, using the slip boundary conditions. The classical solutions for Newtonian fluid in the hydrodynamic case appear as a limiting case of our solutions. The velocity profiles of the flows are presented and the effect of various parameters on velocity is discussed. The results indicate that the presence of couple stresses decreases the velocity of the fluid.
\end{abstract}

\section{Introduction}

The inadequacy of the classical Navier-Stokes theory for describing rheological complex fluids has led to the development of several theories of non-Newtonian fluids. NonNewtonian fluid flows play important role in several industrial manufacturing processes. Few such examples include drilling mud, polymer solutions or melts, and certain oils and greases as well as many other emulsions. Due to the prominent applications in the modern technology and industries, many researchers made attempt to study different non-Newtonian fluid flow problems. One of the popular non-Newtonian fluid models which attracted the interest of several researchers in fluid mechanics is the couple stress fluid model proposed by Stokes [1]. Couple stress fluid model is a simple generalization of the classical Newtonian fluid model that allows the sustenance of couple stresses and body couples in the fluid medium. This theory completely describes the possible effects of couple stresses assuming that the fluid has no microstructure at the kinematical level, so that the kinematics of motion is fully determined by the velocity field [2]. The concept of couple stresses arises due to the way in which the mechanical interactions in the fluid medium are modeled and the stress tensor for this fluid is not symmetric. The couple stress fluid theory has many industrial and scientific applications and can be used to model the flow of synthetic fluids, polymer thickened oils, liquid crystals, animal blood, and synovial fluid present in synovial joints $[2,3]$. It has interesting applications in the theory of lubrication. Numerous researchers have studied the hydrodynamic lubrication of squeeze film flows considering the lubricant to be couple stress fluid and found that the couple stress fluid increases the load carrying capacity of the journal bearing (see Naduvinamani et al. [4], Naduvinamani et al. [5, 6], and Lin and Hung [7] and the references therein).

The study of flows of non-Newtonian fluids with slip boundary condition has gained considerable attention in the recent past. Though many flow problems concerning Newtonian and several non-Newtonian fluids have been solved under the no-slip condition, the fluid slippage might occur at the solid boundary [8-11]. Several investigations indicate the existence of slip at the solid boundary $[12,13]$. As a matter of fact, long back Navier [14] proposed a general boundary condition that permits the possibility of fluid slip at 
a solid boundary. This boundary condition assumes that the tangential velocity of the fluid relative to the solid at a point on its surface is proportional to the tangential stress acting at that point. An excellent review of experimental studies regarding the phenomenon of slip of Newtonian fluids at solid interface is given by Neto et al. [15].

Due to its vast applications in engineering and industry, pressure driven flow or the Poiseuille flow has attracted attention of various researchers. Although pressure driven flows are unidirectional and have been studied earlier for Newtonian and some non-Newtonian fluids, they still attract special attention in a number of emerging problems [1619]. Ellahi [11] has examined the effect of the slip condition on flow of an Oldroyd 8-constant fluid in a channel. Chen and Zhu [20] obtained the analytical solution of CouettePoiseuille flow of Bingham fluids between two porous parallel plates with slip conditions. Wang and $\mathrm{Ng}$ [21] have studied slip flow due to a stretching cylinder. Song and Chen [22] investigated the Poiseuille flow of simple fluids in cylindrical nanochannels. Yang and Zhu [23] have analyzed the squeeze flow of Bingham fluid in the small gap between parallel disks with the Navier slip condition. Ferrás et al. [24] presented analytical solutions of both Newtonian and inelastic nonNewtonian fluids with slip boundary conditions for Couette and Poiseuille flows. Hron et al. [25] established closed form analytical solution for the flows of incompressible non-Newtonian fluids with Navier's slip conditions at the boundary. Ellahi et al. [26] have obtained the exact solutions of nonlinear problems of steady flow of third grade fluid between the concentric cylinders. To the best knowledge of the authors, nobody has attempted to find the analytical solutions of the Poiseuille, Couette, and generalized Couette flows of couple stress fluid between concentric cylinders subject to slip boundary conditions.

The aim of this paper is to establish the closed form analytical solutions for Poiseuille flow, Couette flow, and generalized Couette flows of an incompressible couple stress fluid between two concentric circular cylinders with slip boundary condition. The slip boundary conditions are applied at the boundaries of the inner and outer cylinders. Three cases have been discussed: in the first case it is assumed that both inner and outer cylinders are at rest and the flow is due to the constant pressure gradient which is also known as Poiseuille flow, in the second case the inner cylinder is at rest and the outer cylinder is moving with translatory velocity assuming the pressure is constant (the resultant flow is known as Couette flow), and in the last case it is assumed that the flow is generated due to the translatory motion of the outer cylinder when the inner cylinder is at rest and simultaneously a constant pressure gradient is applied. The flow in the last case is known to be generalized Couette flow or Couette-Poiseuille flow. The paper is organized in terms of seven sections. The next section is devoted to formulating the basic equations governing the flow of couple stress fluid in cylindrical polar coordinates for the presently considered flows. Poiseuille, Couette, and generalized Couette flows are investigated in Sections 3, 4, and 5, respectively, Section 6 is devoted for discussion of results, and the last section contains the concluding remarks.

\section{Basic Equations Governing the Flow}

The equations governing the flow of a couple stress fluid are given by [1]

$$
\begin{gathered}
\frac{d \rho}{d t}+\rho \nabla \cdot \bar{q}=0 \\
\rho \frac{d \bar{q}}{d t}=\rho \bar{f}+\frac{1}{2} \nabla \times(\rho \bar{c})-\nabla p-\mu \nabla \times \nabla \times \bar{q} \\
-\eta \nabla \times \nabla \times \nabla \times \nabla \times \bar{q}+\left(\lambda_{1}+2 \mu\right) \nabla(\nabla \cdot \bar{q}),
\end{gathered}
$$

where $\bar{q}$ and $\rho$ are the velocity and the density of the fluid, respectively, $p$ is the fluid pressure at any point, and $\bar{f}$ and $\bar{c}$ are the body force per unit mass and body couple per unit mass, respectively.

The constitutive equation connecting the force stress tensor $t_{i j}$ and rate of deformation tensor $d_{i j}$ is given by

$$
t_{i j}=\left(-p+\lambda_{1} \nabla \cdot \bar{q}\right) \delta_{i j}+2 \mu d_{i j}-\frac{1}{2} \varepsilon_{i j k}\left[m_{, k}+4 \eta \omega_{k, r r}+\rho c_{r}\right] .
$$

The couple stress tensor $m_{i j}$ that arises in the theory has the linear constitutive relation

$$
m_{i j}=\frac{1}{3} m \delta_{i j}+4 \eta^{\prime} \omega_{j, i}+4 \eta \omega_{i, j}
$$

In the above $\omega=(1 / 2) \nabla \times \bar{q}$ is the spin vector, $\omega_{i, j}$ is the spin tensor, $d_{i j}$ is the rate of deformation tensor, $m$ is the trace of couple stress tensor $m_{i j}$, and $\rho c_{k}$ is the body couple vector. The quantities $\lambda_{1}$ and $\mu$ are the viscosity coefficients and $\eta$ and $\eta^{\prime}$ are the couple stress viscosity coefficients. These material constants are constrained by the inequalities

$$
\mu \geq 0 ; \quad 2 \mu+3 \lambda_{1} \geq 0 ; \quad \eta \geq 0 ; \quad\left|\eta^{\prime}\right| \leq \eta .
$$

In the absence of body forces and body couples, the field equations governing the flow of an incompressible couples stress fluid are

$$
\begin{gathered}
\nabla \cdot \bar{q}=0, \\
\rho \frac{d \bar{q}}{d t}=-\nabla p-\mu \nabla \times \nabla \times \bar{q}-\eta \nabla \times \nabla \times \nabla \times \nabla \times \bar{q} .
\end{gathered}
$$

Here, note that, in the absence of couple stresses (i.e., as $\eta \rightarrow 0$ ), momentum equation (7) reduces to the NavierStokes equation of motion for classical viscous Newtonian fluid.

For unidirectional fully developed steady flow between two concentric horizontal circular cylinders, the velocity field is in the form $\bar{q}=(0,0, w(r))$ and it automatically satisfies continuity equation (6). The momentum equation (7) governing the flow reduces to

$$
\eta \nabla^{4} w-\mu \nabla^{2} w+\frac{\partial p}{\partial z}=0
$$

where $\nabla^{2}=d^{2} / d r^{2}+(1 / r)(d / d r)$. 
In view of the higher order nature of governing equation (8), additional boundary conditions are required to find the solution. In addition to the slip boundary conditions, we use Stokes [2] boundary conditions to solve the governing equation of the flow under consideration. The Stokes boundary condition assumes that the couple stresses vanish on the boundary of the solid.

\section{Fully Developed Flow Generated by the Applied Constant Pressure Gradient (Poiseuille Flow)}

Consider the fully developed steady flow of an incompressible couple stress fluid between two concentric circular cylinders due to the application of constant pressure gradient in the positive $z$-direction. The radiuses of the inner and outer cylinders are, respectively, $R_{1}$ and $R$, and the cylinders are assumed to be at rest. With these, governing equation (8) is to be solved subject to the following slip boundary conditions:

$$
\begin{gathered}
w(R)-\gamma\left[\frac{d}{d r}\left(w-\frac{\eta}{\mu} \nabla^{2} w\right)\right]_{r=R}=0, \\
w\left(R_{1}\right)+\gamma\left[\frac{d}{d r}\left(w-\frac{\eta}{\mu} \nabla^{2} w\right)\right]_{r=R_{1}}=0,
\end{gathered}
$$

$\frac{d^{2} w}{d r^{2}}=0 \quad$ at $r=R, R_{1} \quad$ (Vanishing of couple stresses),

where $R>R_{1}$.

Introducing the following nondimensional parameters

$$
w=\frac{\mu}{\rho R} \bar{w} ; \quad r=R \bar{r} ; \quad p=\frac{\mu^{2}}{\rho R^{2}} \bar{p} ; \quad z=R \bar{z},
$$

momentum equation (8) and boundary conditions (9)-(11), after dropping “-”s, become

$$
\nabla^{2}\left(\nabla^{2}-\frac{1}{a^{2}}\right) w=\frac{G}{a^{2}}
$$

with the boundary conditions

$$
\begin{aligned}
& w(1)-\alpha\left[\frac{d}{d r}\left(w-a^{2} \nabla^{2} w\right)\right]_{r=1}=0, \\
& w(k)+\alpha\left[\frac{d}{d r}\left(w-a^{2} \nabla^{2} w\right)\right]_{r=k}=0,
\end{aligned}
$$

$\frac{d^{2} w}{d r^{2}}=0 \quad$ at $r=1, k \quad$ (Vanishing of couple stresses),

where $a^{2}=\eta / \mu R^{2}, k=R_{1} / R<1, G=-\partial p / \partial z$, and $\alpha=\gamma / R$.

3.1. The Methodology to Find the Solution of the Governing Partial Differential Equation (13). Consider the linear homogeneous differential equation $L w=0$, where $L$ is the differential operator.
Suppose $L$ can be written as $L=L_{1} L_{2}$, where $L_{1}$ and $L_{2}$ are lower order linear operators than $L$.

If $w_{1}$ is a solution of the differential equation $L_{1} w=0$ and $w_{2}$ is a solution of the differential equation $L_{2} w=0$, then, in general, $w_{1}+w_{2}$ need not be a solution of the differential equation $L w=0$.

However, for the governing equation (13) the associated homogeneous differential equation is $\nabla^{2}\left(\nabla^{2}-1 / a^{2}\right) w=0$.

Thus the operator $L$ in this case is $L=\nabla^{2}\left(\nabla^{2}-1 / a^{2}\right)$ in which $L_{1}=\nabla^{2}$ and $L_{2}=\nabla^{2}-1 / a^{2}$.

Therefore, $L_{2}=L_{1}-1 / a^{2}$.

With this, one can easily show that $w_{1}+w_{2}$ is a solution of the differential equation $L w=0$ or $\nabla^{2}\left(\nabla^{2}-1 / a^{2}\right) w=0$.

Further, the solution of the differential equation $\nabla^{2} w=0$ is $w_{1}(r)=A \ln (r)+B$ and the solution of the differential equation $\left(\nabla^{2}-1 / a^{2}\right) w=0$ is $w_{2}(r)=C I_{0}(r / a)+D K_{0}(r / a)$ (because the second operator gives a Bessel differential equation).

Hence, the solution of the homogeneous differential equation $\nabla^{2}\left(\nabla^{2}-1 / a^{2}\right) w=0$ becomes $w(r)=A \ln (r)+B+$ $C I_{0}(r / a)+D K_{0}(r / a)$, which is the complementary function for (13).

The above complementary function with the particular integral will give the solution of the nonhomogeneous partial differential equation (13).

Using the above methodology, the solution of the boundary value problem (13)-(16) is obtained as

$$
w(r)=A_{1} \ln (r)+B_{1}+C_{1} I_{0}\left(\frac{r}{a}\right)+D_{1} K_{0}\left(\frac{r}{a}\right)-\frac{G r^{2}}{4},
$$

where

$$
\begin{aligned}
A_{1}=\frac{1}{D_{55}}\{ & \left(\frac{G k^{2} \alpha}{2 a^{3}}(1+k)-\frac{G k^{2}}{4 a^{3}}\left(1-k^{2}\right)-\frac{G k^{2}}{2 a}\right) \\
& \times\left[I_{0}\left(\frac{k}{a}\right) K_{1}\left(\frac{1}{a}\right)+I_{1}\left(\frac{1}{a}\right) K_{0}\left(\frac{k}{a}\right)\right] \\
& +\left(\frac{G k^{2}}{4 a^{4}}\left(k^{2}-1\right)+\frac{G k^{2} \alpha}{2 a^{4}}(1+k)\right) \\
& \times\left[I_{0}\left(\frac{k}{a}\right) K_{0}\left(\frac{1}{a}\right)-I_{0}\left(\frac{1}{a}\right) K_{0}\left(\frac{k}{a}\right)\right] \\
& +\left(\frac{G k}{4 a^{2}}\left(k^{2}-1\right)+\frac{G k \alpha}{2 a^{2}}(1+k)\right) \\
& \times\left[I_{1}\left(\frac{1}{a}\right) K_{1}\left(\frac{k}{a}\right)-I_{1}\left(\frac{k}{a}\right) K_{1}\left(\frac{1}{a}\right)\right] \\
& -\left(\frac{G k}{4 a^{3}}\left(k^{2}-1\right)+\frac{G k \alpha}{2 a^{3}}(1+k)+\frac{G k}{2 a}\right) \\
& \times\left[I_{1}\left(\frac{k}{a}\right) K_{0}\left(\frac{1}{a}\right)+I_{0}\left(\frac{1}{a}\right) K_{1}\left(\frac{1}{a}\right)\right] \\
& \left.+\frac{G k^{2}}{2}+\frac{G}{2}\right\},
\end{aligned}
$$




$$
\begin{aligned}
& B_{1}=\frac{1}{D_{55}}\left\{I_{0}\left(\frac{k}{a}\right) K_{0}\left(\frac{1}{a}\right)\right. \\
& +I_{1}\left(\frac{k}{a}\right) K_{1}\left(\frac{1}{a}\right) \\
& \times\left[-\frac{G k^{2}}{2 a^{2}} \ln k-\left(k^{2}-a^{2}\right)\right. \\
& \times\left[\left(-\frac{G k \alpha}{2}-\frac{G k}{4 a^{2}}+\frac{G \alpha k}{2 a^{2}}\left(1+a^{2}\right)\right) \ln k\right. \\
& \times\left(\frac{G \alpha}{2 a^{2} k}-\frac{G \alpha}{4 a^{4} k}+\frac{G \alpha^{2}}{2 a^{4} k}\right) \\
& -\frac{G \alpha}{4 a^{2}}+\frac{G \alpha^{2}}{2 a^{2}}+\frac{G k^{3} \alpha}{4 a^{2}}\left(a^{2}-1\right) \\
& +\left(\frac{G k^{2}}{4 a^{4}}-\frac{G k^{2} \alpha}{2 a^{4}}\right) \ln k-\frac{G k^{4} \alpha}{4 a^{4}}\left(a^{2}-1\right) \\
& +\left(\frac{G \alpha^{2} k}{2 a^{4}}-\frac{G \alpha k}{2 a^{2}}\right)\left(k^{2}+a^{2}\right)-\left(1+\frac{\alpha}{k}\right) \\
& \times\left(\frac{G}{2}+\frac{G k^{2} \alpha}{2 a^{2}}-\frac{G k^{2}}{2}-\frac{G}{4 a^{2}}-\frac{G \alpha}{2 a^{2}}\right) \\
& \left.-\frac{G k^{4}}{4 a^{2}}(1-\alpha)\right] \\
& +I_{0}\left(\frac{k}{a}\right) K_{1}\left(\frac{1}{a}\right) \\
& \times\left[\left(-\frac{G \alpha^{2}}{2 a^{3} k}+\frac{G \alpha}{4 a^{3} k}\right)\left(k^{2}-a^{2}\right)\right. \\
& +\left(\frac{G k^{2}}{4 a^{3}}-\frac{G k^{2} \alpha}{2 a^{3}}\right) \ln k-\frac{G k^{4} \alpha}{4 a^{3}}\left(a^{2}-1\right) \\
& -\left(\frac{G \alpha^{2} k}{2 a}-\frac{G \alpha k}{2 a^{2}}-\frac{G \alpha^{2} k}{2 a^{3}}\right)\left(k^{2}+a^{2}\right) \\
& \left.+\left(1+\frac{\alpha}{k}\right)\left(\frac{G}{4 a}-\frac{G k^{2} \alpha}{2 a}-\frac{G \alpha}{2 a}\right)+\frac{G k^{4} \alpha}{4 a}\right] \\
& +I_{0}\left(\frac{k}{a}\right) K_{1}\left(\frac{k}{a}\right) \\
& \times\left[-\frac{G \alpha^{2}}{a}+\left(1+\frac{\alpha}{k}\right)\left(-\frac{G k}{4 a}+\frac{G k \alpha}{a}\right)+\frac{G \alpha}{4 a}\right] \\
& +I_{1}\left(\frac{k}{a}\right) K_{0}\left(\frac{1}{a}\right) \\
& \times\left[\left(\frac{G k}{2 a}(1-\alpha)-\frac{G k}{4 a^{3}}+\frac{G k \alpha}{2 a}\left(a^{2}+1\right)\right) \ln k\right. \\
& +\left(k^{2}-a^{2}\right)\left(\frac{G \alpha}{2 a k^{2}}-\frac{G \alpha}{4 a^{3} k^{2}}+\frac{G \alpha^{2}}{2 a^{3} k^{2}}\right) \\
& +\left(\frac{G k^{3} \alpha}{4 a^{3}}-\frac{G \alpha^{2}}{2 a}\right)\left(a^{2}-1\right) \\
& +\left(-\frac{G \alpha^{2}}{2 a^{3}}+\frac{G \alpha}{2 a^{2}}\right) \\
& \times\left(k^{2}+a^{2}\right)\left(\frac{G a \alpha}{2 k^{2}}-\frac{G \alpha a}{2}+\frac{G k^{3}}{4 a}\right)(1-\alpha) \\
& \left.-\frac{G \alpha}{4 a k^{2}}+\frac{G \alpha^{2}}{2 a k^{2}}\left(1+a^{2}\right)\right] \\
& \left.-\frac{G k^{2} \alpha^{2}}{2 a^{2}}-\frac{G k^{3} \alpha}{4}\right] \\
& +I_{1}\left(\frac{k}{a}\right) K_{0}\left(\frac{k}{a}\right)\left[\frac{G k \alpha}{a}\left(1+\frac{\alpha}{k}\right)-\frac{G \alpha^{2}}{a}-\frac{G k}{4 a}\right] \\
& +I_{0}\left(\frac{1}{a}\right) K_{1}\left(\frac{1}{a}\right)\left[-\frac{G k^{2}}{2 a} \ln k-\frac{G k \alpha}{a}-\frac{G k^{2}}{4 a}\right] \\
& +I_{0}\left(\frac{1}{a}\right) K_{0}\left(\frac{k}{a}\right) \\
& \times\left[\left(-\frac{G}{4 a^{2}}+\frac{G k^{2} \alpha}{2 a^{2}}+\frac{G \alpha}{2 a^{2}}\right)\left(1+\frac{\alpha}{k}\right)\right. \\
& -\left(\frac{G \alpha}{2 a^{2} k}-\frac{G \alpha}{4 a^{4} k}+\frac{G \alpha^{2}}{2 a^{4} k}\right)\left(k^{2}-a^{2}\right) \\
& +\left(\frac{G k^{2}}{2 a^{2}}+\frac{G k^{2} \alpha}{2 a^{4}}-\frac{G k^{2}}{4 a^{4}}\right) \ln k-\frac{G k^{4} \alpha}{4 a^{4}} \\
& \left.-\frac{G \alpha^{2} k}{2 a^{4}}\left(k^{2}+a^{2}\right)-\frac{G k^{2}}{2}+\frac{G k^{4}}{4 a^{2}}+\frac{G k^{3} \alpha}{2 a^{2}}\right] \\
& +I_{0}\left(\frac{1}{a}\right) K_{1}\left(\frac{k}{a}\right) \\
& \times\left[\left(-\frac{G \alpha}{4 a^{3} k^{2}}+\frac{G \alpha^{2}}{2 a^{3} k^{2}}\right)\left(k^{2}-a^{2}\right)\right. \\
& +\left(\frac{G k \alpha}{2 a^{3}}-\frac{G k}{4 a^{3}}+\frac{G k}{2 a}\right) \ln k \\
& -\frac{G k^{3} \alpha}{4 a^{3}}-\frac{G k^{2} \alpha^{2}}{2 a^{3}}+\frac{G \alpha}{2 a}-\frac{G \alpha^{2} a}{2 k^{2}}-\frac{G a \alpha}{2} \\
& +\frac{G k^{3}}{4 a}+\frac{G \alpha}{2 a}\left(k^{2}+a^{2}\right)-\frac{G \alpha}{4 a k^{2}} \\
& \left.+\frac{G \alpha^{2}}{2 a k^{2}}\left(1+a^{2}\right)\right] \\
& +I_{1}\left(\frac{1}{a}\right) K_{0}\left(\frac{1}{a}\right)\left[-\frac{G k^{2}}{2 a} \ln k-\frac{G k \alpha}{a}-\frac{G k^{2}}{2 a}\right] \\
& +I_{1}\left(\frac{1}{a}\right) K_{0}\left(\frac{k}{a}\right) \\
& \times\left[\left(1+\frac{\alpha}{k}\right)\left(\frac{G}{4 a}-\frac{G k^{2} \alpha}{2 a}-\frac{G \alpha}{2 a}\right)+\frac{G k^{4} \alpha}{4 a}\right. \\
& +\left(\frac{G \alpha}{4 a^{3} k}-\frac{G \alpha^{2}}{2 a^{3} k}\right)\left(k^{2}-a^{2}\right)
\end{aligned}
$$




$$
\begin{aligned}
& +\left(\frac{G k^{2}}{4 a^{3}}-\frac{G k^{2} \alpha}{2 a^{3}}\right) \ln k-\frac{G k^{4} \alpha}{4 a^{3}}\left(a^{2}-1\right) \\
& \left.+\frac{G \alpha^{2} k}{2 a^{3}}\left(k^{2}+a^{2}\right)\right] \\
& +I_{1}\left(\frac{1}{a}\right) K_{1}\left(\frac{k}{a}\right) \\
& \times\left[\frac{G \alpha}{4 a^{2}}+\left(-\frac{G k \alpha}{2 a^{2}}+\frac{G k}{4 a^{2}}\right) \ln k\right. \\
& \left.\left.-\frac{G \alpha^{2}}{2 a^{2}}+\frac{G k^{3} \alpha}{4 a^{2}}+\frac{G k^{2} \alpha^{2}}{2 a^{2}}\right]\right\}, \\
& C_{1}=\frac{1}{D_{55}}\left\{K _ { 0 } ( \frac { 1 } { a } ) \left[\frac{G}{2}\left(1-k^{2}\right)+\frac{G}{4 a^{2}}\left(k^{2}-1\right)\right.\right. \\
& \left.+\frac{G \alpha}{a^{2}}(0.5+k)+\frac{G k^{2}}{2 a^{2}}(\alpha+\ln k)\right] \\
& +K_{1}\left(\frac{1}{a}\right)\left[\frac{G}{4 a}\left(k^{2}-1\right)+\frac{G \alpha}{2 a}(1+2 k)\right. \\
& \left.+\frac{G k^{2}}{2 a}(\alpha+\ln k)\right] \\
& +K_{0}\left(\frac{k}{a}\right)\left[\frac{G}{2}\left(k^{2}-1\right)-\frac{G k^{2}}{4 a^{2}}\left(k^{2}-1\right)\right. \\
& \left.-\frac{G \alpha k^{2}}{a^{2}}(0.5 k+1)-\frac{G k}{2 a^{2}}(\alpha+k \ln k)\right] \\
& +K_{1}\left(\frac{k}{a}\right)\left[-\frac{G \alpha k^{2}}{2 a}-\frac{G \alpha k}{a}-\frac{G}{2 a}(\alpha+k \ln k)\right. \\
& \left.\left.-\frac{G k}{4 a}\left(k^{2}-1\right)\right]\right\} \\
& D_{1}=\frac{1}{D_{55}}\left\{I _ { 0 } ( \frac { 1 } { a } ) \left[\frac{G}{2}\left(k^{2}-1\right)-\frac{G}{4 a^{2}}\left(k^{2}-1\right)\right.\right. \\
& \left.-\frac{G \alpha}{a^{2}}(0.5+k)-\frac{G k^{2}}{2 a^{2}}(\alpha+\ln k)\right] \\
& +I_{1}\left(\frac{1}{a}\right)\left[\frac{G}{4 a}\left(k^{2}-1\right)+\frac{G \alpha}{2 a}(1+2 k)\right. \\
& \left.+\frac{G k^{2}}{2 a}(\alpha+\ln k)\right] \\
& +I_{0}\left(\frac{k}{a}\right)\left[\frac{G}{2}\left(1-k^{2}\right)+\frac{G k^{2}}{4 a^{2}}\left(k^{2}-1\right)\right. \\
& \left.+\frac{G \alpha k^{2}}{2 a^{2}}(k+2)+\frac{G k}{2 a^{2}}(\alpha+k \ln k)\right] \\
& +I_{1}\left(\frac{k}{a}\right)\left[-\frac{G \alpha}{a}(0.5+k)-\frac{G k}{2 a}(k \alpha+\ln k)\right. \\
& \left.\left.-\frac{G k}{4 a}\left(k^{2}-1\right)\right]\right\} \text {, }
\end{aligned}
$$

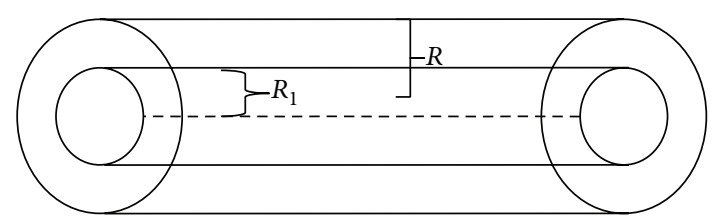

FIGURE 1: Flow geometry.

$$
\begin{aligned}
D_{55}= & {\left[I_{1}\left(\frac{1}{a}\right) K_{1}\left(\frac{k}{a}\right)-I_{1}\left(\frac{k}{a}\right) K_{1}\left(\frac{1}{a}\right)\right] } \\
& \times\left[\frac{k \alpha}{a^{2}}+\frac{k}{a^{2}} \ln k+\frac{\alpha}{a^{2}}\right] \\
& +\left[I_{1}\left(\frac{1}{a}\right) K_{0}\left(\frac{k}{a}\right)+I_{0}\left(\frac{k}{a}\right) K_{1}\left(\frac{1}{a}\right)\right] \\
& \times\left[\frac{k^{2} \alpha}{a^{3}}+\frac{k^{2}}{a^{3}} \ln k+\frac{\alpha k}{a^{3}}+\frac{1}{a}\right] \\
& -\left[I_{0}\left(\frac{1}{a}\right) K_{1}\left(\frac{k}{a}\right)+I_{1}\left(\frac{k}{a}\right) K_{0}\left(\frac{1}{a}\right)\right] \\
& \times\left[\frac{k \alpha}{a^{3}}+\frac{k}{a^{3}} \ln k+\frac{\alpha}{a^{3}}-\frac{k}{a}\right] \\
& +\left[I_{0}\left(\frac{k}{a}\right) K_{0}\left(\frac{1}{a}\right)-I_{0}\left(\frac{1}{a}\right) K_{0}\left(\frac{k}{a}\right)\right] \\
& \times\left[\frac{k^{2} \alpha}{a^{4}}+\frac{k^{2}}{a^{4}} \ln k+\frac{\alpha k}{a^{4}}+\frac{1}{a^{2}}-\frac{k^{2}}{a^{2}}\right]-2 .
\end{aligned}
$$

\section{Fully Developed Flow Generated by the Translatory Motion of the Outer Cylinder (Couette Flow)}

Here, the flow of an incompressible couple stress fluid between two concentric circular cylinders is generated due to the translatory motion of the outer cylinder with constant velocity $(0,0, U)$ while the inner cylinder is assumed to be at rest. The flow geometry is as shown in Figure 1. Assuming that the pressure in the flow region is constant, the governing equation (8) takes the form

$$
\eta \nabla^{4} w-\mu \nabla^{2} w=0
$$

with the boundary conditions

$$
\begin{gathered}
w(R)-\gamma\left[\frac{d}{d r}\left(w-\frac{\eta}{\mu} \nabla^{2} w\right)\right]_{r=R}=U, \\
w\left(R_{1}\right)+\gamma\left[\frac{d}{d r}\left(w-\frac{\eta}{\mu} \nabla^{2} w\right)\right]_{r=R_{1}}=0,
\end{gathered}
$$

$\frac{d^{2} w}{d r^{2}}=0 \quad$ at $r=R, R_{1} \quad$ (Vanishing of couple stresses),

where $R>R_{1}$. 
Using the nondimensional variables

$$
w=U \bar{w} ; \quad r=R \bar{r} ; \quad z=R \bar{z}
$$

the boundary value problem (19)-(22), after dropping “-”s, becomes

$$
\nabla^{2}\left(\nabla^{2}-\frac{1}{a^{2}}\right) w=0
$$

with the boundary conditions

$$
\begin{aligned}
& w(1)-\alpha\left[\frac{d}{d r}\left(w-a^{2} \nabla^{2} w\right)\right]_{r=1}=1, \\
& w(k)+\alpha\left[\frac{d}{d r}\left(w-a^{2} \nabla^{2} w\right)\right]_{r=k}=0,
\end{aligned}
$$

$\frac{d^{2} w}{d r^{2}}=0 \quad$ at $r=1, k \quad$ (Vanishing of couple stresses),

where $a^{2}=\eta / \mu R^{2}, k=R_{1} / R<1$, and $\alpha=\gamma / R$.

Now, using the methodology described earlier, the exact solution of boundary value problem (24)-(27) which is valid for the entire flow field is given by

$$
w(r)=A_{2} \ln (r)+B_{2}+C_{2} I_{0}\left(\frac{r}{a}\right)+D_{2} K_{0}\left(\frac{r}{a}\right)
$$

where

$$
\begin{aligned}
& A_{2}=\frac{1}{D_{55}} \\
& \times\left\{\frac { k ^ { 2 } } { a ^ { 4 } } \left[\frac{a^{2}}{k}\left(K_{1}\left(\frac{1}{a}\right) I_{1}\left(\frac{k}{a}\right)-K_{1}\left(\frac{k}{a}\right) I_{1}\left(\frac{1}{a}\right)\right)\right.\right. \\
& -a\left(K_{1}\left(\frac{1}{a}\right) I_{0}\left(\frac{k}{a}\right)+K_{0}\left(\frac{k}{a}\right) I_{1}\left(\frac{1}{a}\right)\right) \\
& +\frac{a}{k}\left(K_{0}\left(\frac{1}{a}\right) I_{1}\left(\frac{k}{a}\right)-K_{1}\left(\frac{k}{a}\right) I_{0}\left(\frac{1}{a}\right)\right) \\
& \left.\left.+K_{0}\left(\frac{k}{a}\right) I_{0}\left(\frac{1}{a}\right)-K_{0}\left(\frac{1}{a}\right) I_{0}\left(\frac{k}{a}\right)\right]\right\}, \\
& C_{2}=\frac{1}{D_{55}}\left[\frac{1}{a}\left(k K_{1}\left(\frac{k}{a}\right)-K_{1}\left(\frac{1}{a}\right)\right)\right. \\
& \left.-\frac{1}{a^{2}}\left(k^{2} K_{0}\left(\frac{k}{a}\right)-K_{0}\left(\frac{1}{a}\right)\right)\right], \\
& D_{2}=\frac{1}{D_{55}}\left[\frac{1}{a}\left(k I_{1}\left(\frac{k}{a}\right)-I_{1}\left(\frac{1}{a}\right)\right)\right. \\
& \left.-\frac{1}{a^{2}}\left(k^{2} I_{0}\left(\frac{k}{a}\right)-I_{0}\left(\frac{1}{a}\right)\right)\right],
\end{aligned}
$$

$$
\begin{aligned}
& B_{2}=\frac{1}{D_{55}}\left\{\left(\frac{k^{2}}{a^{4}} \ln k+\frac{\alpha k}{a^{4}}\right)\right. \\
& \times\left[\frac{a^{2}}{k}\left(K_{1}\left(\frac{k}{a}\right) I_{1}\left(\frac{1}{a}\right)-K_{1}\left(\frac{1}{a}\right) I_{1}\left(\frac{k}{a}\right)\right)\right. \\
& +a\left(K_{1}\left(\frac{1}{a}\right) I_{0}\left(\frac{k}{a}\right)+K_{0}\left(\frac{k}{a}\right) I_{1}\left(\frac{1}{a}\right)\right) \\
& -\frac{a}{k}\left(K_{0}\left(\frac{1}{a}\right) I_{1}\left(\frac{k}{a}\right)+K_{1}\left(\frac{k}{a}\right) I_{0}\left(\frac{1}{a}\right)\right) \\
& \left.+K_{0}\left(\frac{1}{a}\right) I_{0}\left(\frac{k}{a}\right)-K_{0}\left(\frac{k}{a}\right) I_{0}\left(\frac{1}{a}\right)\right] \\
& +\frac{1}{a}\left(K_{1}\left(\frac{1}{a}\right) I_{0}\left(\frac{k}{a}\right)+K_{0}\left(\frac{k}{a}\right) I_{1}\left(\frac{1}{a}\right)\right) \\
& +\frac{1}{a^{2}}\left(K_{0}\left(\frac{1}{a}\right) I_{0}\left(\frac{k}{a}\right)\right. \\
& \left.\left.-K_{0}\left(\frac{k}{a}\right) I_{0}\left(\frac{1}{a}\right)\right)-1\right\} \text {, } \\
& D_{55}=\left[I_{1}\left(\frac{1}{a}\right) K_{1}\left(\frac{k}{a}\right)-I_{1}\left(\frac{k}{a}\right) K_{1}\left(\frac{1}{a}\right)\right] \\
& \times\left[\frac{k \alpha}{a^{2}}+\frac{k}{a^{2}} \ln k+\frac{\alpha}{a^{2}}\right] \\
& +\left[I_{1}\left(\frac{1}{a}\right) K_{0}\left(\frac{k}{a}\right)+I_{0}\left(\frac{k}{a}\right) K_{1}\left(\frac{1}{a}\right)\right] \\
& \times\left[\frac{k^{2} \alpha}{a^{3}}+\frac{k^{2}}{a^{3}} \ln k+\frac{\alpha k}{a^{3}}+\frac{1}{a}\right] \\
& -\left[I_{0}\left(\frac{1}{a}\right) K_{1}\left(\frac{k}{a}\right)+I_{1}\left(\frac{k}{a}\right) K_{0}\left(\frac{1}{a}\right)\right] \\
& \times\left[\frac{k \alpha}{a^{3}}+\frac{k}{a^{3}} \ln k+\frac{\alpha}{a^{3}}-\frac{k}{a}\right] \\
& +\left[I_{0}\left(\frac{k}{a}\right) K_{0}\left(\frac{1}{a}\right)-I_{0}\left(\frac{1}{a}\right) K_{0}\left(\frac{k}{a}\right)\right] \\
& \times\left[\frac{k^{2} \alpha}{a^{4}}+\frac{k^{2}}{a^{4}} \ln k+\frac{\alpha k}{a^{4}}+\frac{1}{a^{2}}-\frac{k^{2}}{a^{2}}\right]-2 .
\end{aligned}
$$

\section{Generalized Couette Flow}

Here again, the physical model is similar to that of the earlier two problems. The flow is generated due to the translatory motion of the outer cylinder with constant velocity $(0,0, U)$ while the inner cylinder is at rest and simultaneously a constant pressure gradient is applied in the positive $z$ direction. The momentum equation governing this flow is

$$
\eta \nabla^{4} w-\mu \nabla^{2} w-G=0
$$


subject to the conditions

$$
\begin{aligned}
& w(R)-\gamma\left[\frac{d}{d r}\left(w-\frac{\eta}{\mu} \nabla^{2} w\right)\right]_{r=R}=U, \\
& w\left(R_{1}\right)+\gamma\left[\frac{d}{d r}\left(w-\frac{\eta}{\mu} \nabla^{2} w\right)\right]_{r=R_{1}}=0
\end{aligned}
$$

$\frac{d^{2} w}{d r^{2}}=0 \quad$ at $r=R, R_{1} \quad$ (Vanishing of couple stresses),

where $R>R_{1}$.

With the nondimensional parameters

$$
w=U \bar{w} ; \quad r=R \bar{r} ; \quad p=\frac{\mu U}{R} \bar{p} ; \quad z=R \bar{z},
$$

(30)-(33), after dropping “-” s, become

$$
\nabla^{2}\left(\nabla^{2}-\frac{1}{a^{2}}\right) w=\frac{G}{a^{2}},
$$

with the boundary conditions

$$
\begin{aligned}
& w(1)-\alpha\left[\frac{d}{d r}\left(w-a^{2} \nabla^{2} w\right)\right]_{r=1}=1, \\
& w(k)+\alpha\left[\frac{d}{d r}\left(w-a^{2} \nabla^{2} w\right)\right]_{r=k}=0,
\end{aligned}
$$

$\frac{d^{2} w}{d r^{2}}=0 \quad$ at $r=1, k \quad$ (Vanishing of couple stresses),

where $G=-\partial p / \partial z, a^{2}=\eta / \mu R^{2}, k=R_{1} / R<1$, and $\alpha=\gamma / R$.

Employing the procedure described in Section 3, the analytical solution of the boundary value problem (35)-(38) which is valid for the entire flow field is given by

$$
w(r)=A_{3} \ln (r)+B_{3}+C_{3} I_{0}\left(\frac{r}{a}\right)+D_{3} K_{0}\left(\frac{r}{a}\right)-\frac{G r^{2}}{4},
$$

where

$$
\begin{aligned}
A_{3}=\frac{1}{D_{55}}\left\{K_{0}\left(\frac{1}{a}\right) I_{1}\left(\frac{k}{a}\right)\right. \\
\times\left[\frac{G k}{2 a}(\alpha-1)-\frac{G k}{4 a^{3}}\left(k^{2}-1\right)\right. \\
\left.\quad-\frac{G \alpha k}{2 a^{3}}\left(1+a^{2}+k\right)+\frac{k}{a^{3}}\right] \\
+K_{0}\left(\frac{1}{a}\right) I_{0}\left(\frac{k}{a}\right)
\end{aligned}
$$

$$
\begin{aligned}
& \times\left[\frac{G k^{2}}{4 a^{4}}\left(k^{2}-1\right)+\frac{G \alpha k^{2}}{2 a^{4}}(1+k)-\frac{k^{2}}{a^{4}}\right] \\
& +K_{1}\left(\frac{1}{a}\right) I_{1}\left(\frac{k}{a}\right) \\
& \times\left[\frac{G k}{4 a^{2}}\left(1-k^{2}\right)-\frac{G k \alpha}{2 a^{2}}(1+k)+\frac{k}{a^{2}}\right] \\
& +K_{1}\left(\frac{1}{a}\right) I_{0}\left(\frac{k}{a}\right) \\
& \times\left[-\frac{G \alpha k^{2}}{2 a}+\frac{G k^{2}}{4 a^{3}}\left(k^{2}-1\right)\right. \\
& \left.+\frac{G \alpha k^{2}}{2 a^{3}}\left(1+a^{2}+k\right)-\frac{G k^{2}}{2 a}-\frac{k^{2}}{a^{3}}\right] \\
& +K_{0}\left(\frac{k}{a}\right) I_{1}\left(\frac{1}{a}\right) \\
& \times\left[-\frac{G k^{2}}{2 a}+\frac{G k^{2}}{4 a^{3}}\left(k^{2}-1\right)\right. \\
& \left.+\frac{G \alpha k^{2}}{2 a^{3}}\left(1+a^{2}+k\right)-\frac{G \alpha k^{2}}{2 a}-\frac{k^{2}}{a^{3}}\right] \\
& -K_{0}\left(\frac{k}{a}\right) I_{0}\left(\frac{1}{a}\right) \\
& \times\left[\frac{G k^{2}}{4 a^{4}}\left(k^{2}-1\right)+\frac{G \alpha k^{2}}{2 a^{4}}(1+k)-\frac{k^{2}}{a^{4}}\right] \\
& -K_{1}\left(\frac{k}{a}\right) I_{1}\left(\frac{1}{a}\right) \\
& \times\left[\frac{G k}{4 a^{2}}\left(1-k^{2}\right)-\frac{G k \alpha}{2 a^{2}}(1+k)+\frac{k}{a^{2}}\right] \\
& +K_{1}\left(\frac{k}{a}\right) I_{0}\left(\frac{1}{a}\right) \\
& \times\left[\frac{G k}{2 a}(\alpha-1)-\frac{G k}{4 a^{3}}\left(k^{2}-1\right)\right. \\
& \left.\left.-\frac{G \alpha k}{2 a^{3}}\left(1+a^{2}+k\right)+\frac{k}{a^{3}}\right]+\frac{G k^{2}}{2}+\frac{G}{2}\right\}, \\
& D_{3}=\frac{1}{D_{55}}\left\{I _ { 0 } ( \frac { 1 } { a } ) \left[\frac{G}{2}\left(k^{2}-1\right)-\frac{G}{4 a^{2}}\left(k^{2}-1\right)\right.\right. \\
& \left.-\frac{G \alpha}{a^{2}}(0.5+k)+\frac{1}{a^{2}}-\frac{G k^{2}}{2 a^{2}}(\alpha+\ln k)\right] \\
& +I_{1}\left(\frac{1}{a}\right)\left[\frac{G}{4 a}\left(k^{2}-1\right)+\frac{G \alpha}{2 a}(1+2 k)\right. \\
& \left.-\frac{1}{a}+\frac{G k^{2}}{2 a}(\alpha+\ln k)\right] \\
& +I_{0}\left(\frac{k}{a}\right)\left[\frac{G}{2}\left(1-k^{2}\right)+\frac{G k^{2}}{4 a^{2}}\left(k^{2}-1\right)\right.
\end{aligned}
$$




$$
\begin{aligned}
& +\frac{G \alpha k^{2}}{2 a^{2}}(k+2)-\frac{k^{2}}{a^{2}} \\
& \left.+\frac{G k}{2 a^{2}}(\alpha+k \ln k)\right] \\
& +I_{1}\left(\frac{k}{a}\right)\left[-\frac{G \alpha}{a}(0.5+k)+\frac{k}{a}\right. \\
& \left.\left.-\frac{G k}{2 a}(k \alpha+\ln k)-\frac{G k}{4 a}\left(k^{2}-1\right)\right]\right\}, \\
& C_{3}=\frac{1}{D_{55}}\left\{K _ { 0 } ( \frac { 1 } { a } ) \left[\frac{G}{2}\left(1-k^{2}\right)+\frac{G}{4 a^{2}}\left(k^{2}-1\right)\right.\right. \\
& \left.+\frac{G \alpha}{a^{2}}(0.5+k)-\frac{1}{a^{2}}+\frac{G k^{2}}{2 a^{2}}(\alpha+\ln k)\right] \\
& +K_{1}\left(\frac{1}{a}\right)\left[\frac{G}{4 a}\left(k^{2}-1\right)+\frac{G \alpha}{2 a}(1+2 k)\right. \\
& \left.-\frac{1}{a}+\frac{G k^{2}}{2 a}(\alpha+\ln k)\right] \\
& +K_{0}\left(\frac{k}{a}\right)\left[\frac{G}{2}\left(k^{2}-1\right)-\frac{G k^{2}}{4 a^{2}}\left(k^{2}-1\right)\right. \\
& -\frac{G \alpha k^{2}}{2 a^{2}}(k+1) \\
& \left.+\frac{k^{2}}{a^{2}}-\frac{G \alpha k^{2}}{2 a^{2}}-\frac{G k}{2 a^{2}}(\alpha+k \ln k)\right] \\
& +K_{1}\left(\frac{k}{a}\right)\left[-\frac{G \alpha k^{2}}{2 a}-\frac{G \alpha k}{a}+\frac{k}{a}\right. \\
& \left.\left.-\frac{G}{2 a}(\alpha+k \ln k)-\frac{G k}{4 a}\left(k^{2}-1\right)\right]\right\}, \\
& D_{55}=\left[I_{1}\left(\frac{1}{a}\right) K_{1}\left(\frac{k}{a}\right)-I_{1}\left(\frac{k}{a}\right) K_{1}\left(\frac{1}{a}\right)\right] \\
& \times\left[\frac{k \alpha}{a^{2}}+\frac{k}{a^{2}} \ln k+\frac{\alpha}{a^{2}}\right] \\
& +\left[I_{1}\left(\frac{1}{a}\right) K_{0}\left(\frac{k}{a}\right)+I_{0}\left(\frac{k}{a}\right) K_{1}\left(\frac{1}{a}\right)\right] \\
& \times\left[\frac{k^{2} \alpha}{a^{3}}+\frac{k^{2}}{a^{3}} \ln k+\frac{\alpha k}{a^{3}}+\frac{1}{a}\right] \\
& -\left[I_{0}\left(\frac{1}{a}\right) K_{1}\left(\frac{k}{a}\right)+I_{1}\left(\frac{k}{a}\right) K_{0}\left(\frac{1}{a}\right)\right] \\
& \times\left[\frac{k \alpha}{a^{3}}+\frac{k}{a^{3}} \ln k+\frac{\alpha}{a^{3}}-\frac{k}{a}\right] \\
& +\left[I_{0}\left(\frac{k}{a}\right) K_{0}\left(\frac{1}{a}\right)-I_{0}\left(\frac{1}{a}\right) K_{0}\left(\frac{k}{a}\right)\right] \\
& \times\left[\frac{k^{2} \alpha}{a^{4}}+\frac{k^{2}}{a^{4}} \ln k+\frac{\alpha k}{a^{4}}+\frac{1}{a^{2}}-\frac{k^{2}}{a^{2}}\right]-2, \\
& B_{3}=\frac{1}{D_{55}}\left\{I_{1}\left(\frac{k}{a}\right) K_{0}\left(\frac{k}{a}\right)\right. \\
& \times\left[\frac{G k \alpha}{2 a}\left(1+\frac{\alpha}{k}-\frac{a^{2} \alpha}{k}\right)\right. \\
& \left.-\frac{k}{a}\left(1+\frac{G}{4}-\frac{G \alpha}{2}\right)+\frac{G \alpha}{2 a}\right] \\
& +I_{1}\left(\frac{1}{a}\right) K_{0}\left(\frac{k}{a}\right) \\
& \times\left[\left(1+\frac{\alpha}{k}\right)\right. \\
& \times\left(\frac{1}{a}+\frac{G}{4 a}-\frac{G \alpha}{2 a}-\frac{G k^{2} \alpha}{2 a}\right) \\
& +\frac{k^{2}}{a^{3}}\left(1+\frac{G}{4}-\frac{G \alpha}{2}\right)\left(\ln k+\frac{\alpha}{k^{3}}\left(k^{2}-a^{2}\right)\right) \\
& \left.+\frac{k^{2} \alpha}{a^{3}}\left(\frac{G k^{2}}{4}+\frac{G k \alpha}{2}+\frac{G a^{2} \alpha}{2 k}\right)\right] \\
& +I_{0}\left(\frac{1}{a}\right) K_{0}\left(\frac{k}{a}\right) \\
& \times\left[\frac{G k^{2} \alpha}{2 a^{2}}\left(1+\frac{\alpha}{k}-\frac{a^{2} \alpha}{k}-a^{2}\right)-\left(1+\frac{\alpha}{k}\right)\right. \\
& \times\left(\frac{1}{a^{2}}+\frac{G}{4 a^{2}}-\frac{G \alpha}{2}-\frac{G \alpha}{2 a^{2}}-\frac{G}{2}+\frac{G k^{2}}{2}\right) \\
& -\frac{k^{2}}{a^{4}}\left(1+\frac{G}{4}-\frac{G \alpha}{2}\right)\left(\ln k+\frac{\alpha}{k^{3}}\left(k^{2}-a^{2}\right)\right) \\
& -\frac{k^{2} \alpha}{a^{4}}\left(\frac{G k^{2}}{4}+\frac{G k \alpha}{2}+\frac{G a^{2} \alpha}{2 k}\right) \\
& +\frac{k^{2}}{a^{2}}\left(\frac{G k^{2}}{4}+\frac{G k \alpha}{2}+\frac{G a^{2} \alpha}{2 k}\right) \\
& +\frac{G k^{2}}{2 a^{2}}\left(\ln k+\frac{\alpha}{k^{3}}\left(k^{2}-a^{2}\right)\right) \\
& \left.+\left(\alpha+\frac{\alpha^{2}}{k}\right)\left(-\frac{G}{2}+\frac{G k^{2}}{2}\right)\right] \\
& +I_{0}\left(\frac{k}{a}\right) K_{1}\left(\frac{k}{a}\right) \\
& \times\left[\frac{\alpha}{a}\left(1+\frac{G}{4}-\frac{G \alpha}{2}\right)-\frac{G \alpha^{2}}{2 a}\right. \\
& \left.+\left(1+\frac{\alpha}{k}\right)\left(-\frac{G k}{4 a}+\frac{G k \alpha}{a}-\frac{k}{a}\right)\right] \\
& +I_{1}\left(\frac{1}{a}\right) K_{1}\left(\frac{k}{a}\right) \\
& \times\left[-\frac{G \alpha^{2}}{2 a}+\frac{\alpha}{k^{2}}\left(1+\frac{G}{4}-\frac{G \alpha}{2}\right)\right.
\end{aligned}
$$




$$
\begin{aligned}
& +k \alpha\left(\frac{G k^{2}}{4}+\frac{G k \alpha}{2}+\frac{G a^{2} \alpha}{2 k}\right) \\
& +\frac{k}{a^{2}}\left(1+\frac{G}{4}-\frac{G \alpha}{2}\right)\left(\ln k+\frac{\alpha}{k^{3}}\left(k^{2}-a^{2}\right)\right) \\
& \left.-\frac{k \alpha}{a^{2}}\left(a^{2}-1\right)\left(\frac{G k^{2}}{2}+\frac{G k \alpha}{2}+\frac{G a^{2} \alpha}{2 k}\right)\right] \\
& +I_{0}\left(\frac{1}{a}\right) K_{1}\left(\frac{k}{a}\right) \\
& \times\left[\frac{G \alpha^{2}}{2 a}\left(1-a^{2}\right)-\frac{\alpha}{a k^{2}}\left(1+\frac{G}{4}-\frac{G \alpha}{2}+\frac{G a^{2} \alpha}{2}\right)\right. \\
& +(1-\alpha)\left(\frac{G k^{3}}{4 a}+\frac{G k^{2} \alpha}{2 a}\right) \\
& -\frac{k}{a^{3}}\left(1+\frac{G}{4}-\frac{G \alpha}{2}\right)\left(\ln k+\frac{\alpha}{k^{3}}\left(k^{2}-a^{2}\right)\right) \\
& +\frac{k \alpha}{a^{3}}\left(a^{2}-1\right)\left(\frac{G k^{2}}{4}+\frac{G k \alpha}{2}+\frac{G a^{2} \alpha}{2 k}\right) \\
& \left.+\frac{G k}{2 a}\left(\ln k+\frac{\alpha}{k^{3}}\left(k^{2}-a^{2}\right)\right)+(1-\alpha) \frac{G a \alpha}{2 k^{2}}\right] \\
& +I_{1}\left(\frac{k}{a}\right) K_{0}\left(\frac{1}{a}\right) \\
& \times\left[(1-\alpha) \frac{G k}{2 a} \ln k+\frac{k}{a}\left(\frac{G k^{2}}{4}+\frac{G k \alpha}{2}+\frac{G a^{2} \alpha}{2 k}\right)\right. \\
& +\frac{\alpha}{a k^{2}}\left(-\frac{G}{4}+\frac{G \alpha}{2}+\frac{G a^{2} \alpha}{2}\right) \\
& -\frac{k}{a^{3}}\left(1+\frac{G}{4}-\frac{G \alpha}{2}-\frac{G a^{2} \alpha}{2}\right) \\
& \times\left(\ln k+\frac{\alpha}{k^{3}}\left(k^{2}-a^{2}\right)\right) \\
& -\frac{k \alpha}{a^{3}}\left(\frac{G k^{2}}{4}+\frac{G k \alpha}{2}+\frac{G a^{2} \alpha}{2 k}\right) \\
& -\frac{G \alpha^{2}}{2 a}\left(a^{2}-1\right)-\left(\frac{a \alpha}{k^{2}}-\frac{a \alpha^{2}}{k^{2}}\right) \\
& \times\left(-\frac{G}{2}+\frac{G k^{2}}{2}\right) \\
& \left.+(1-\alpha)\left(k^{2}-a^{2}\right) \frac{G \alpha}{2 a k^{2}}-\frac{\alpha}{a k^{2}}\right] \\
& +I_{0}\left(\frac{k}{a}\right) K_{0}\left(\frac{1}{a}\right) \\
& \times\left[(\alpha-1) \frac{G k^{2}}{2 a^{2}} \ln k\right. \\
& -\frac{k^{2}}{a^{2}}\left(\frac{G k^{2}}{4}+\frac{G k \alpha}{2}+\frac{G a^{2} \alpha}{2 k}\right)
\end{aligned}
$$

$$
\begin{aligned}
& -\left(1+\frac{\alpha}{k}\right)\left(-\frac{G}{4 a^{2}}+\frac{G \alpha}{2}+\frac{G \alpha}{2 a^{2}}\right) \\
& +\left(\frac{G k^{2}}{4 a^{4}}-\frac{G k^{2} \alpha}{2 a^{4}}-\frac{G k^{2} \alpha}{2 a^{2}}+\frac{k^{2}}{a^{4}}\right) \\
& \times\left(\ln k+\frac{\alpha}{k^{3}}\left(k^{2}-a^{2}\right)\right) \\
& +\frac{k^{2} \alpha}{a^{4}}\left(\frac{G k^{2}}{4}+\frac{G a^{2} \alpha}{2}-\frac{G k \alpha}{2 k}\right) \\
& -\frac{G k^{2} \alpha}{2 a^{2}}\left(1+\frac{\alpha}{k}-a^{2}-\frac{a^{2} \alpha}{k}\right) \\
& +\left(1+\frac{\alpha}{k}\right)\left(-\frac{G}{2}+\frac{G k^{2}}{2}\right) \\
& -\left(\alpha+\frac{\alpha^{2}}{k}\right)\left(-\frac{G}{2}+\frac{G k^{2}}{2}\right) \\
& \left.-(1-\alpha)\left(k^{2}-a^{2}\right) \frac{G \alpha}{2 a^{2} k}+\frac{1}{a^{2}}\left(1+\frac{\alpha}{k}\right)\right] \\
& +I_{1}\left(\frac{1}{a}\right) K_{0}\left(\frac{1}{a}\right) \\
& \times\left[-\frac{G k^{2}}{2 a^{2}} \ln k-(1-\alpha)\left(\frac{G k^{2}}{4 a}+\frac{G k \alpha}{2 a}+\frac{G a \alpha}{2 k}\right)\right. \\
& -\left(\frac{G k^{2} \alpha}{4 a}+\frac{G \alpha^{2} k}{2 a}+\frac{G a^{2} \alpha^{2}}{2 a k}\right) \\
& \left.-\left(k^{2}-a^{2}\right) \frac{G \alpha}{2 a k}\right] \\
& +I_{1}\left(\frac{k}{a}\right) K_{1}\left(\frac{1}{a}\right) \\
& \times\left[\frac{G \alpha^{2}}{2}\left(1-a^{2}\right)+\frac{\alpha}{k^{2}}\left(-\frac{G}{4}+\frac{G \alpha}{2}+\frac{G a^{2} \alpha}{2}\right)\right. \\
& -\frac{G \alpha^{2}}{2 k^{2}}\left(k^{2}-a^{2}\right)-\frac{G k \alpha}{2} \ln k \\
& -\left(\frac{G k}{4 a^{2}}-\frac{G \alpha k}{2 a^{2}}-\frac{G k \alpha}{2}+\frac{k}{a^{2}}\right) \\
& \times\left(\ln k+\frac{\alpha}{k^{3}}\left(k^{2}-a^{2}\right)\right) \\
& -\frac{k \alpha}{a^{2}}\left(\frac{G k^{2}}{4}+\frac{G k \alpha}{2 a}+\frac{G a^{2} \alpha}{2 k}\right) \\
& \left.+\frac{a^{2} \alpha^{2}}{k^{2}}\left(-\frac{G}{2}+\frac{G k^{2}}{2}\right)-\frac{\alpha}{k^{2}}\right] \\
& +I_{0}\left(\frac{k}{a}\right) K_{1}\left(\frac{1}{a}\right) \\
& \times\left[\frac{G k^{2} \alpha}{2 a} \ln k-\left(1+\frac{\alpha}{k}\right)\right.
\end{aligned}
$$


TABle 1: Limiting solutions of (i) Poiseuille flow, (ii) Couette flow, and (iii) generalized Couette flow.

\begin{aligned} & (i) $\quad w(r)=\frac{k}{k \alpha+\alpha+k \ln (k)}\left[\ln (r)\left(\frac{G k^{2}}{4}+\frac{G k \alpha}{2}+\frac{G \alpha}{2}-\frac{G}{4}\right)+\left(-\frac{G \alpha}{2}+\frac{G}{4}\right)\left(\ln k+\frac{\alpha}{k}\right)+\alpha\left(\frac{G k^{2}}{4}+\frac{G k \alpha}{2}\right)\right]-\frac{G r^{2}}{4} \\ &$ (ii) $\quad w(r)=\frac{\alpha+k \ln (k / r)}{\alpha+k \alpha+k \ln (k)} \\ &$ (iii) $\quad w(r)=\frac{k}{k \alpha+\alpha+k \ln (k)}\left[\ln (r)\left(\frac{G k^{2}}{4}+\frac{G k \alpha}{2}+\frac{G \alpha}{2}-\frac{G}{4}-1\right)+\left(-\frac{G \alpha}{2}+\frac{G}{4}+1\right)\left(\ln k+\frac{\alpha}{k}\right)+\alpha\left(\frac{G k^{2}}{4}+\frac{G k \alpha}{2}\right)\right]-\frac{G r^{2}}{4} \\ &$\hline\end{aligned}

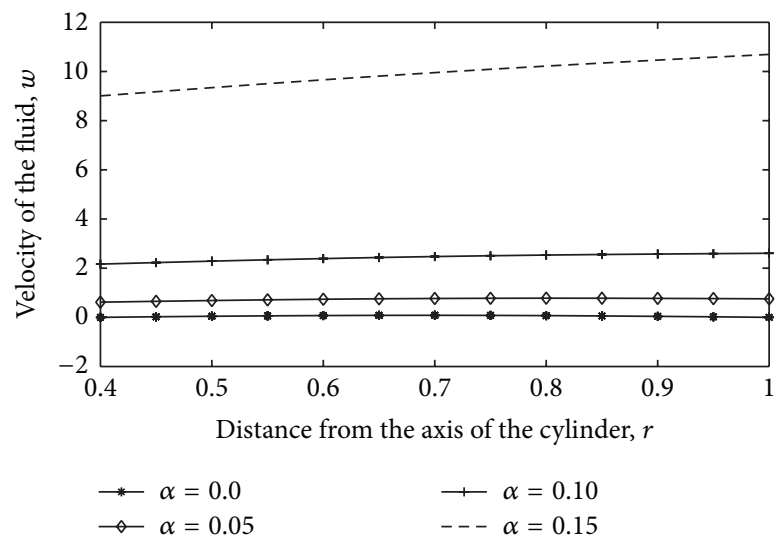

(a) $G=10 ; a=0.4$

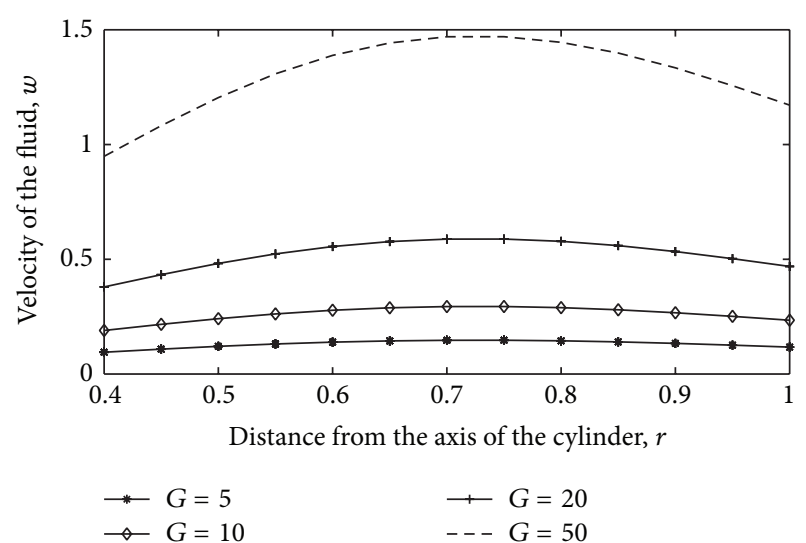

(c) $a=0.4 ; \alpha=0.02$

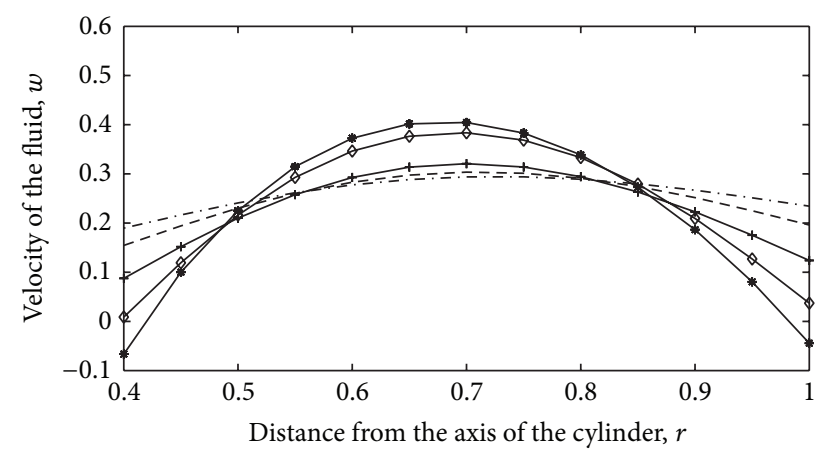
$\rightarrow$ * Newtonian fluid $\quad---a=0.3$
$\rightarrow a=0.1$$$
\text { -. }-a=0.4
$$$$
+a=0.2
$$

(b) $\alpha=0.02 ; G=10$

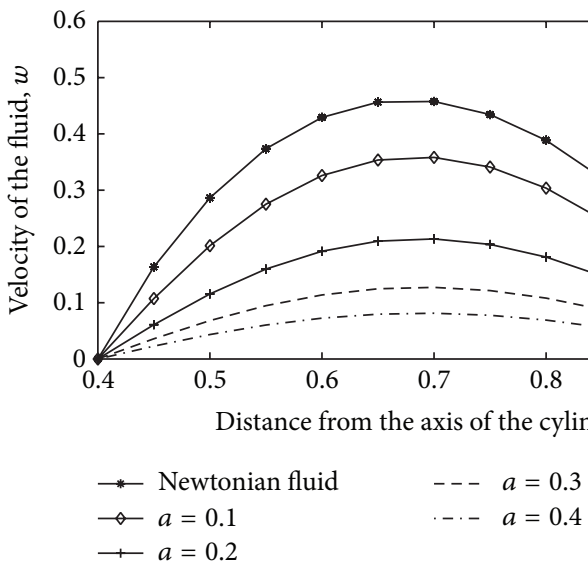

(d) $G=10 ; \alpha=0$ (no-slip boundary condition)

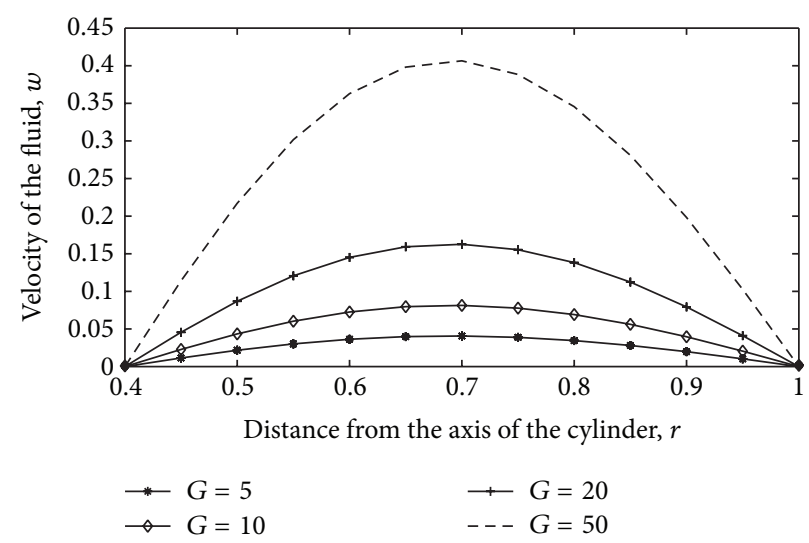

(e) $a=0.4 ; \alpha=0$ (no-slip boundary condition)

FIGURE 2: Velocity profiles for Poiseuille flow. 


$$
\begin{aligned}
& \times\left(-\frac{G}{4 a}+\frac{G \alpha}{2 a}+\frac{G a \alpha}{2}\right) \\
& +\frac{G \alpha^{2}}{2 a k}\left(k^{2}-a^{2}\right) \\
& -\left(\ln k+\frac{\alpha}{k^{3}}\left(k^{2}-a^{2}\right)\right) \\
& \times\left(\frac{G k^{2}}{4 a^{3}}-\frac{G \alpha k^{2}}{2 a^{3}}-\frac{G k^{2} \alpha}{2 a}+\frac{k^{2}}{a^{3}}\right) \\
& +\frac{k^{2} \alpha}{a^{3}}\left(\frac{G k^{2}}{4}+\frac{G k \alpha}{2}+\frac{G a^{2} \alpha}{2 k}\right) \\
& +\frac{G k^{2} \alpha}{2 a}\left(1+\frac{\alpha}{k}\right)\left(a^{2}-1\right) \\
& -\left(1+\frac{\alpha}{k}\right)\left(-\frac{G a \alpha}{2}+\frac{G k^{2} a \alpha}{2}\right) \\
& \left.+\frac{1}{a}\left(1+\frac{\alpha}{k}\right)\right] \\
& +I_{0}\left(\frac{1}{a}\right) K_{1}\left(\frac{1}{a}\right) \\
& \left.\times\left[-\frac{1}{a}\left(\frac{G k^{2}}{4}+\frac{G k \alpha}{2 a}\right)-\frac{G k^{2}}{2 a} \ln k-\frac{G k \alpha}{2 a}\right]\right\} .
\end{aligned}
$$

\section{Discussion of Results}

In all of the three considered problems, the governing equations give rise to Bessel differential equations. The solutions are obtained in terms of Bessel function and are simplified using recurrence relations of Bessel functions [27]. The velocity profiles for different flow parameters are presented and the effect of slip parameter, couple stress parameter, and the pressure gradient on velocity for each of the problems is described. We have the couple stress parameter $a^{2}=\eta / \mu R^{2}$; in the absence of couple stresses, that is, as $\eta \rightarrow 0$, the parameter $a \rightarrow 0$ and the corresponding limiting solutions to the problems considered in the paper are given in Table 1 . It is noted that these limiting solutions are the same as that of the classical solutions for Newtonian fluid.

From Figure 2, it can be observed that the increasing of slip parameter has an increasing effect on the velocity. That is, the more the fluid slips at the boundary, the less its velocity is affected by the motion of the boundary. The graphs also indicate that, as the couple stress parameter $a$ increases, there is a decrease in the velocity. Since $a^{2}=\eta / \mu R^{2}, a$ increases as the couple stress viscosity $\eta$ increases. Thus, increasing of couple stresses has a decreasing effect on the fluid velocity. It can also be seen from the above figures that the velocity of the fluid increases when we increase the pressure gradient.

It can be seen from Figure 3(a) that, for Couette flow, as the slip parameter increases, the velocity decreases near the stationary cylinder while the trend is reversed near the moving cylinder. It is observed from Figure 3(b) that

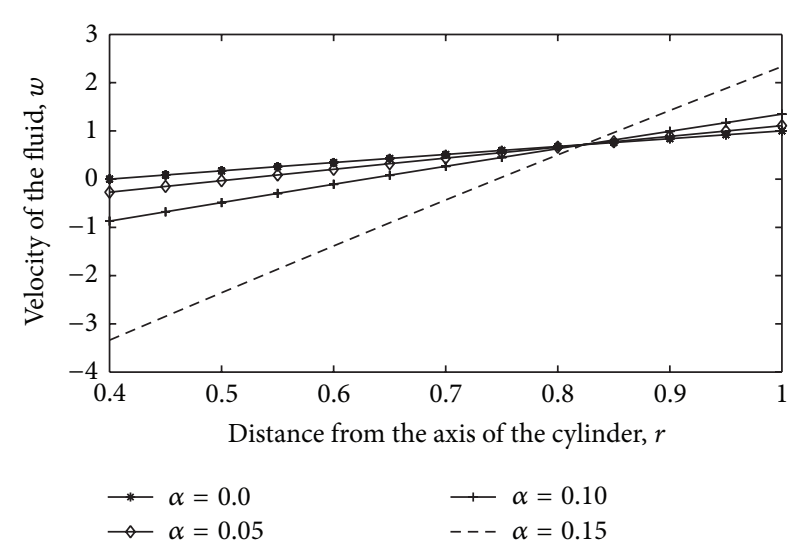

(a) $a=0.4$

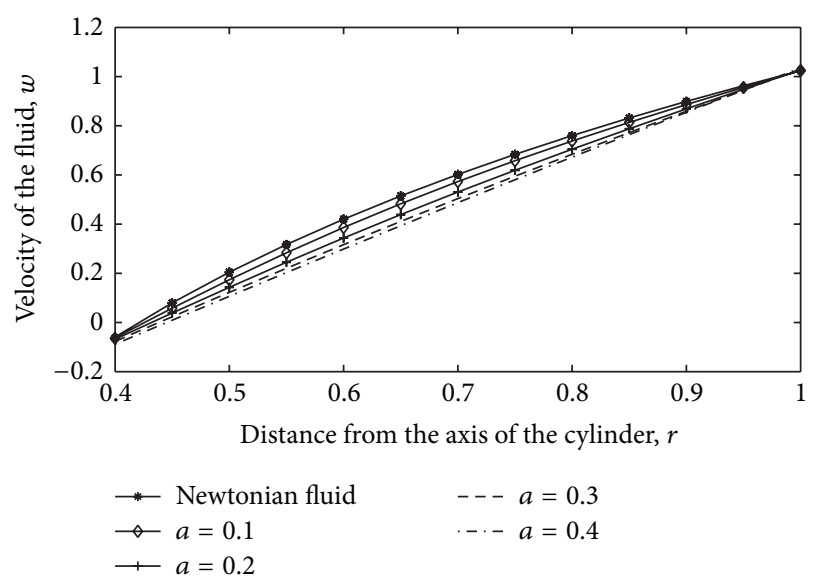

(b) $\alpha=0.02$

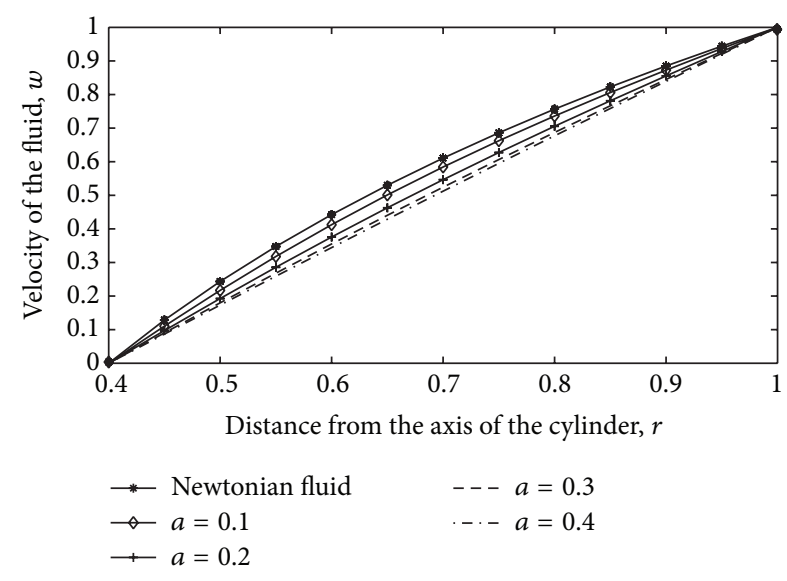

(c) $\alpha=0$ (no-slip boundary condition)

FIGURE 3: Velocity profiles for Couette flow.

increasing of couple stress parameter $a$ has a decreasing effect on the velocity. Moreover, under the slip boundary condition the velocity of the fluid increases from couple stress fluid to Newtonian fluid and a similar trend is seen under the no-slip boundary condition as well.

Here again, it is found that the increasing of couple stress parameter has a decreasing effect on the velocity 


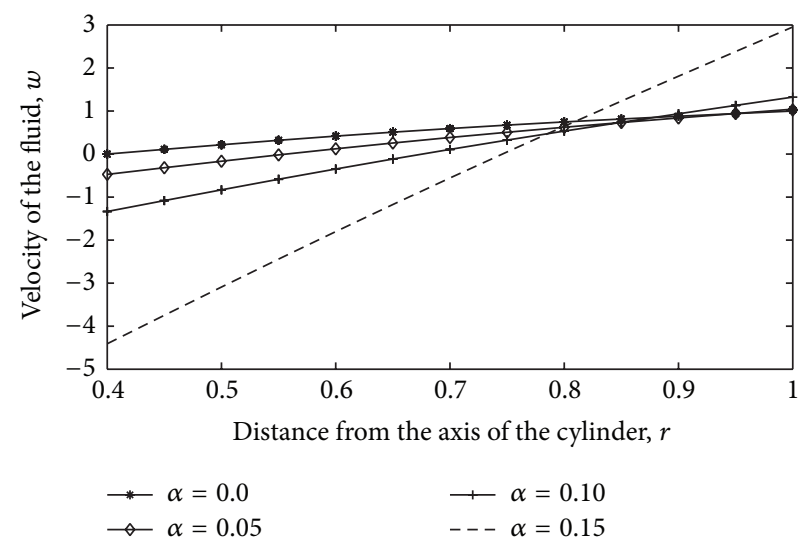

(a) $G=10 ; a=0.4$

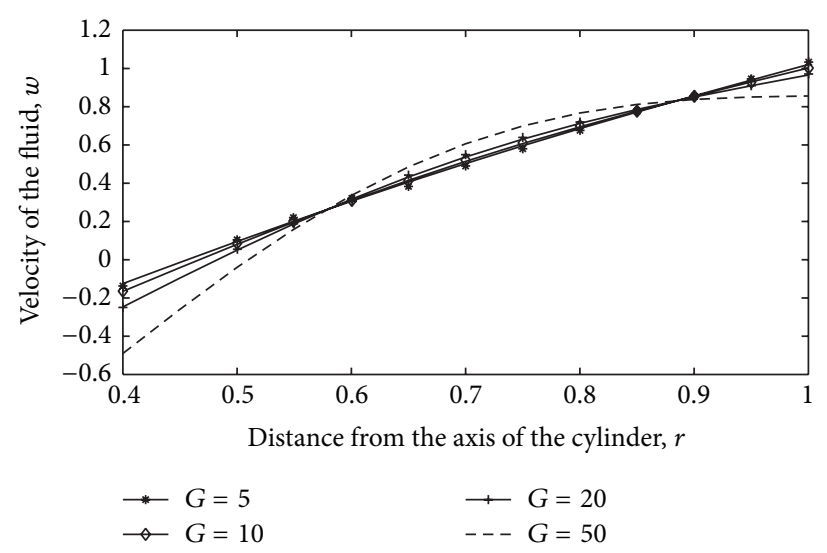

(c) $a=0.4 ; \alpha=0.02$

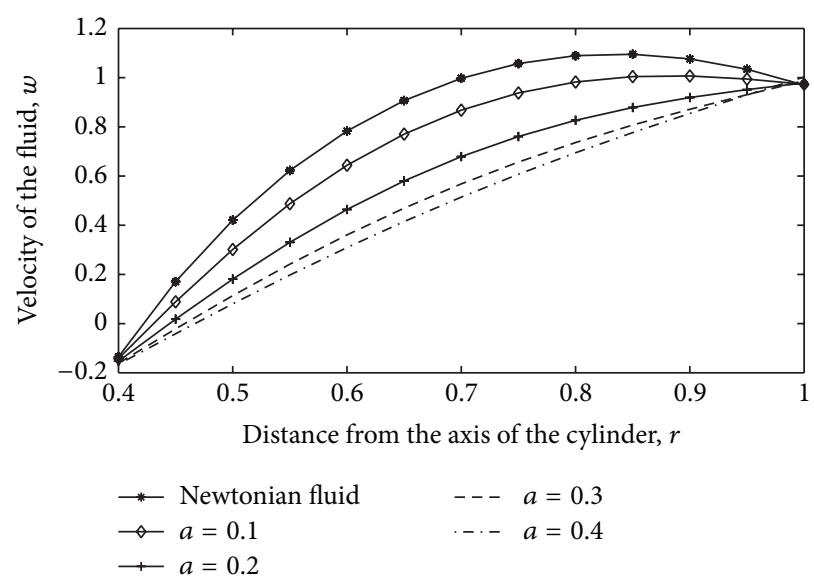

(b) $\alpha=0.02 ; G=10$

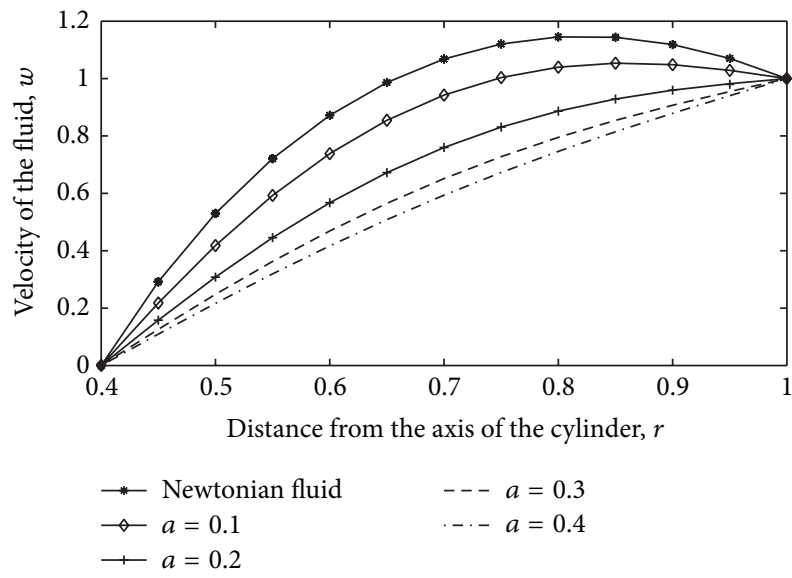

(d) $\alpha=0 ; G=10$, (no-slip boundary condition)

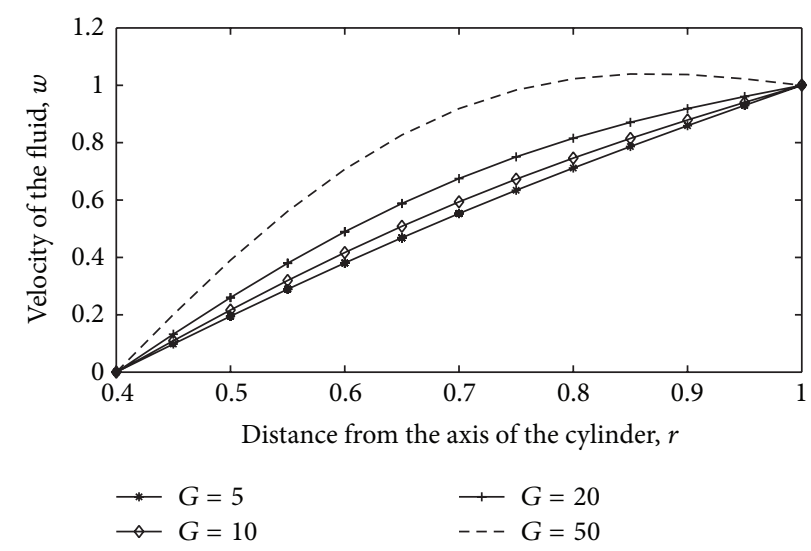

(e) $a=0.4 ; \alpha=0$ (no-slip boundary condition)

FIGURE 4: Velocity profiles for generalized Couette flow.

and as the slip parameter increases, the velocity decreases near the stationary cylinder while the trend is reversed near the moving cylinder (see Figure 4). As expected, the velocity shows increasing trend with the increasing of pressure gradient. It is also seen from Figures $4(\mathrm{a})$ and $4(\mathrm{~d})$ that, under the slip boundary condition, the velocity of the fluid increases gradually from couple stress fluid to
Newtonian fluid when compared to the no-slip boundary condition.

\section{Concluding Remarks}

In this paper, three types of unidirectional fully developed flows of couple stress fluid between two concentric circular 
cylinders are presented, namely, the fully developed flow due to the applied constant pressure gradient, the fully developed flow due to the translatory motion of the outer cylinder, and the flow generated due to the simultaneous application of constant pressure gradient and the translatory motion of the outer cylinder. The closed form analytical expressions of the velocity are obtained in terms of Bessel functions for each of the problems. As expected, increase in the pressure gradient has corresponding increase in the velocity of the fluid. The observations of the present study are the following.

(i) The classical solutions for Newtonian fluid in the hydrodynamic case appear as limiting case of our solutions.

(ii) The presence of couple stresses has a decreasing effect on the fluid velocity.

(iii) The more the fluid slips at the boundary (i.e., as the slip parameter increases), the less its velocity is affected by the boundary.

(iv) Under the slip boundary condition the fluid velocity changes gradually as compared to the no-slip boundary condition.

\section{Conflict of Interests}

The authors declare that there is no conflict of interests regarding the publication of this paper.

\section{References}

[1] V. K. Stokes, "Couple stresses in fluids," Physics of Fluids, vol. 9, no. 9, pp. 1709-1715, 1966.

[2] V. K. Stokes, Theories of Fluids with Microstructure, Springer, New York, NY, USA, 1984.

[3] M. Devakar and T. K. V. Iyengar, "Run up flow of a couple stress fluid between parallel plates," Nonlinear Analysis: Modelling and Control, vol. 15, no. 1, pp. 29-37, 2010.

[4] N. B. Naduvinamani, P. S. Hiremath, and G. Gurubasavaraj, "Effect of surface roughness on the couple-stress squeeze film between a sphere and a flat plate," Tribology International, vol. 38, no. 5, pp. 451-458, 2005.

[5] N. B. Naduvinamani, S. T. Fathima, and P. S. Hiremath, "Hydrodynamic lubrication of rough slider bearings with couple stress fluids," Tribology International, vol. 36, no. 12, pp. 949-959, 2003.

[6] N. B. Naduvinamani, S. T. Fathima, and P. S. Hiremath, "Effect of surface roughness on characteristics of couplestress squeeze film between anisotropic porous rectangular plates," Fluid Dynamics Research, vol. 32, no. 5, pp. 217-231, 2003.

[7] J.-R. Lin and C.-R. Hung, "Combined effects of non-Newtonian couple stresses and fluid inertia on the squeeze film characteristics between a long cylinder and an infinite plate," Fluid Dynamics Research, vol. 39, no. 8, pp. 616-639, 2007.

[8] E. A. Ashmawy, "Unsteady couette flow of a micropolar fluid with slip," Meccanica, vol. 47, no. 1, pp. 85-94, 2012.

[9] P. A. Thompson and S. M. Troian, "A general boundary condition for liquid flow at solid surfaces," Nature, vol. 389, no. 6649, pp. 360-362, 1997.
[10] S. Richardson, “On the no-slip boundary condition," Journal of Fluid Mechanics, vol. 59, pp. 707-719, 1973.

[11] R. Ellahi, "Effects of the slip boundary condition on nonNewtonian flows in a channel," Communications in Nonlinear Science and Numerical Simulation, vol. 14, no. 4, pp. 1377-1384, 2009.

[12] M. E. O.'Neill, K. B. Ranger, and H. Brenner, "Slip at the surface of a translating-rotating sphere bisected by a free surface bounding a semi-infinite viscous fluid: removal of the contactline singularity," Physics of Fluids, vol. 29, pp. 913-924, 1986.

[13] A. B. Basset, A Treatise on Hydrodynamics, vol. 2, Dover, New York, NY, USA, 1961.

[14] C. L. M. H. Navier, "Memoirs de l'Academie," Royale des Sciences de l'Institut de France, vol. 1, pp. 414-416, 1823.

[15] C. Neto, D. R. Evans, E. Bonaccurso, H.-J. Butt, and V. S. J. Craig, "Boundary slip in Newtonian liquids: a review of experimental studies," Reports on Progress in Physics, vol. 68, no. 12, pp. 28592897, 2005.

[16] H. S. Tang, "Analysis on creeping channel flows of compressible fluids subject to wall slip," Rheologica Acta, vol. 51, no. 5, pp. 421439, 2012.

[17] C. Pozrikidis, "Gravity-driven creeping flow of two adjacent layers through a channel and down a plane wall," Journal of Fluid Mechanics, vol. 371, pp. 345-376, 1998.

[18] S. G. Hatzikiriakos and J. M. Dealy, "Wall slip of molten high density polyethylenes. II capillary rheometer studies," Journal of Rheology, vol. 36, pp. 703-741, 1992.

[19] H. S. Tang and D. M. Kalyon, "Estimation of the parameters of Herschel-Bulkley fluid under wall slip using a combination of capillary and squeeze flow viscometers," Rheologica Acta, vol. 43, no. 1, pp. 80-88, 2004.

[20] Y.-L. Chen and K.-Q. Zhu, "Couette-Poiseuille flow of Bingham fluids between two porous parallel plates with slip conditions," Journal of Non-Newtonian Fluid Mechanics, vol. 153, no. 1, pp. $1-11,2008$.

[21] C. Y. Wang and C.-O. Ng, "Slip flow due to a stretching cylinder," International Journal of Non-Linear Mechanics, vol. 46, no. 9, pp. 1191-1194, 2011.

[22] X. Song and J. K. Chen, "A comparative study on poiseuille flow of simple fluids through cylindrical and slit-like nanochannels," International Journal of Heat and Mass Transfer, vol. 51, no. 7-8, pp. 1770-1779, 2008.

[23] S.-P. Yang and K.-Q. Zhu, "Analytical solutions for squeeze flow of Bingham fluid with Navier slip condition," Journal of NonNewtonian Fluid Mechanics, vol. 138, no. 2-3, pp. 173-180, 2006.

[24] L. L. Ferrás, J. M. Nóbrega, and F. T. Pinho, "Analytical solutions for Newtonian and inelastic non-Newtonian flows with wall slip," Journal of Non-Newtonian Fluid Mechanics, vol. 175-176, pp. 76-88, 2012.

[25] J. Hron, C. Le Roux, J. Málek, and K. R. Rajagopal, "Flows of incompressible fluids subject to Navier's slip on the boundary," Computers and Mathematics with Applications, vol. 56, no. 8, pp. 2128-2143, 2008.

[26] R. Ellahi, T. Hayat, F. M. Mahomed, and S. Asghar, "Effects of slip on the non-linear flows of a third grade fluid," Nonlinear Analysis: Real World Applications, vol. 11, no. 1, pp. 139-146, 2010.

[27] N. W. McLachlan, Bessel Functions for Engineers, Oxford Engineering Science Series, 2nd edition, 1961. 


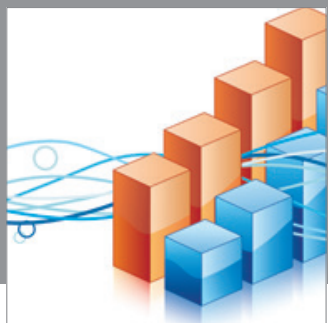

Advances in

Operations Research

mansans

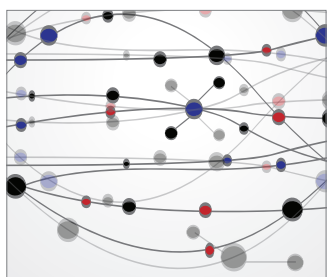

The Scientific World Journal
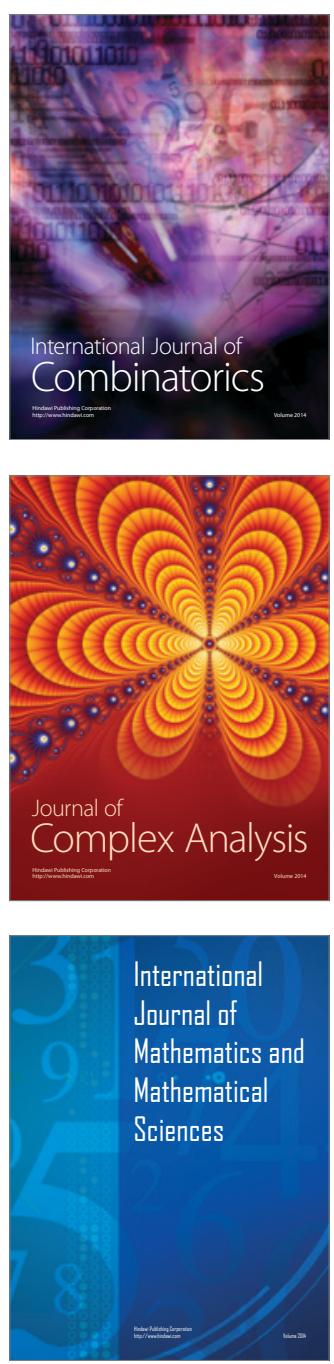
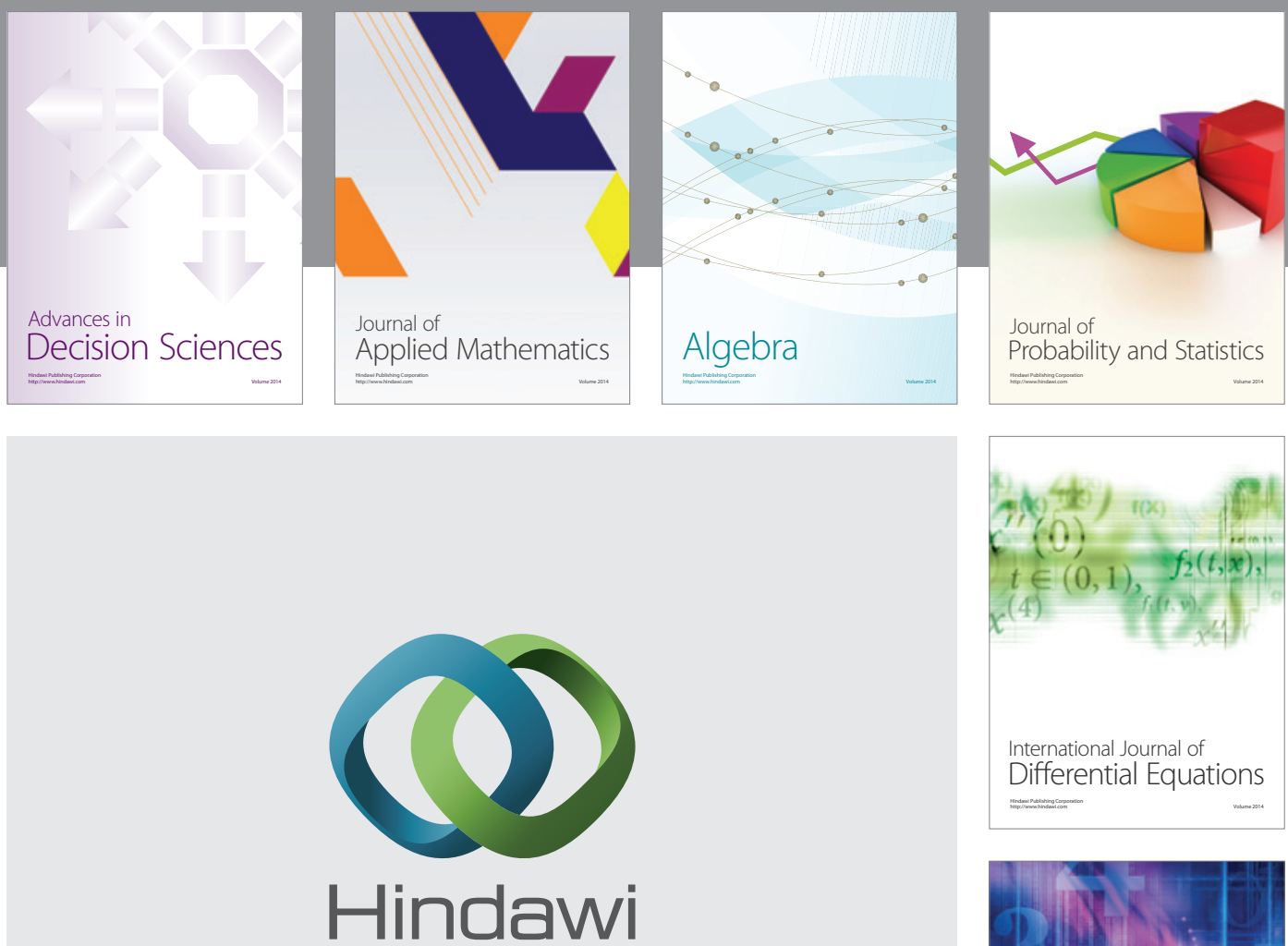

Submit your manuscripts at http://www.hindawi.com
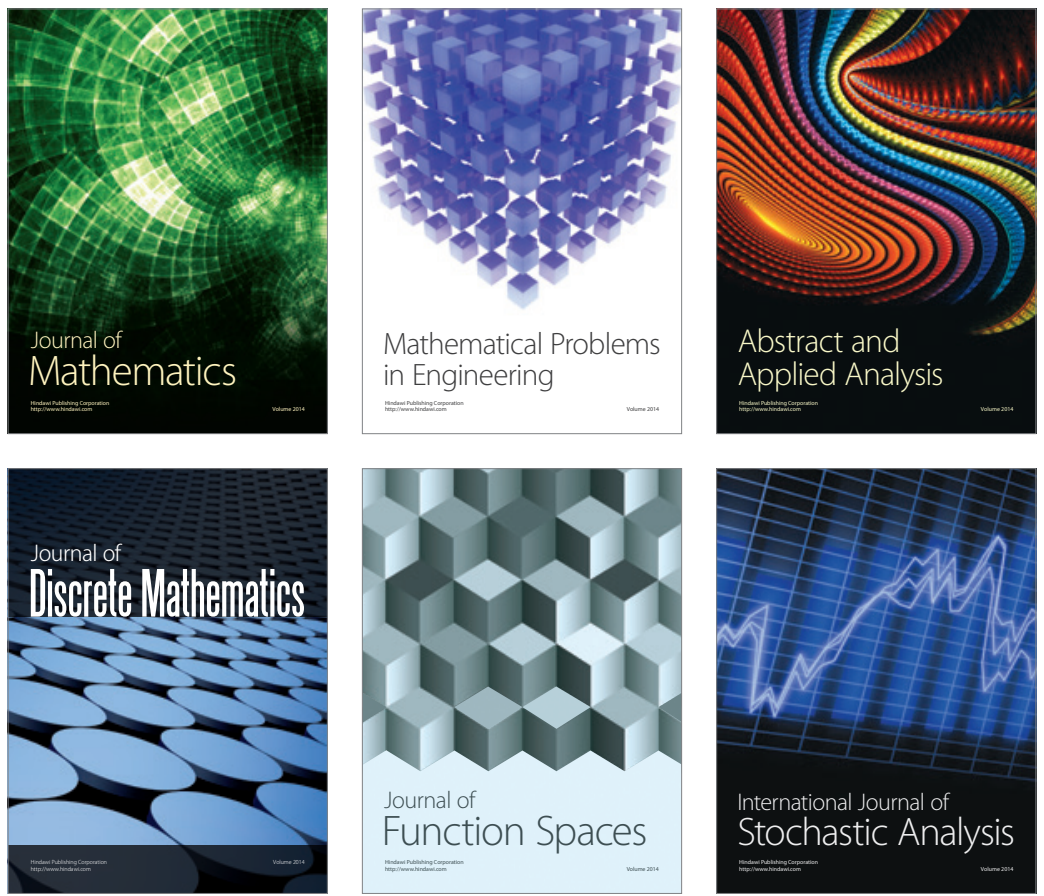

Journal of

Function Spaces

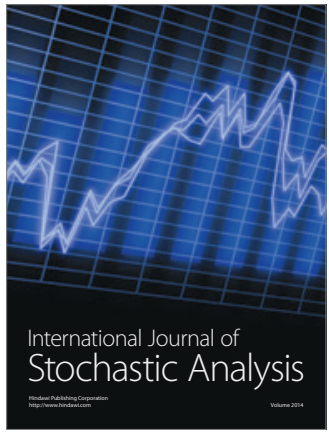

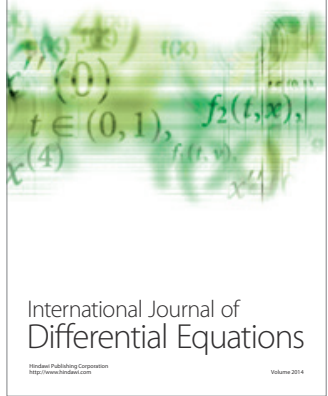
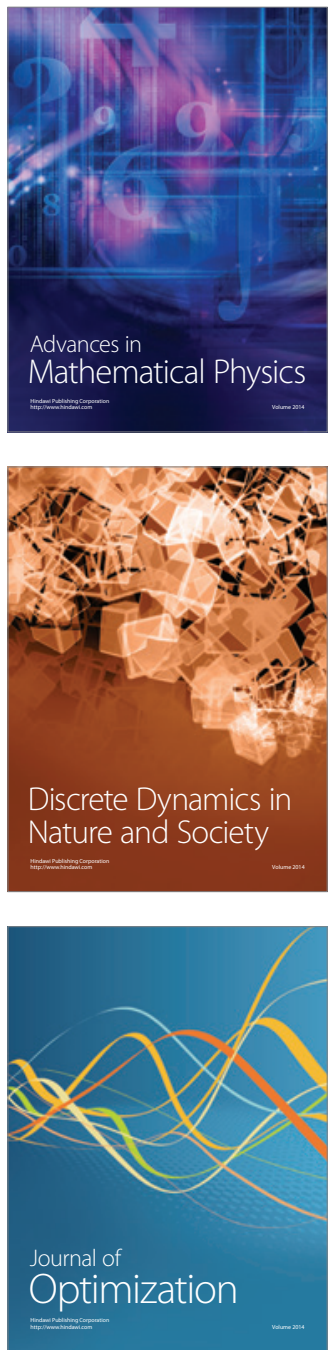